\title{
Genetic diversity of Rhizoctonia solani AG-3 from potato and tobacco in North Carolina
}

\author{
Paulo C. Ceresini \\ Departamento de Biologia, Faculdade de Engenharia, \\ Universidade Estadual Paulista "Júlio de Mesquita \\ Filho" (UNESP), 15385-000, Ilha Solteira, São Paulo, \\ Brazil
}

H. David Shew

Department of Plant Pathology, North Carolina State University, Raleigh, North Carolina 27695

Rytas J. Vilgalys

Department of Biology, Duke University, Durham, North Carolina 27708

Marc A. Cubeta ${ }^{1}$

Department of Plant Pathology, North Carolina State University, Raleigh, North Carolina 27695

\begin{abstract}
Anastomosis group 3 (AG-3) of Rhizoctonia solani (teleomorph $=$ Thanatephorus cucumeris) is frequently associated with diseases of potato (AG-3 PT) and tobacco (AG-3 TB). Although isolates of $R$. solani AG-3 from these two Solanaceous hosts are somatically related based on anastomosis reaction and taxonomically related based on fatty acid, isozyme and DNA characters, considerable differences are evident in their biology, ecology, and epidemiology. However, genetic diversity among field populations of $R$. solani AG-3 PT and TB has not been documented. In this study, the genetic diversity of field populations of $R$. solani AG-3 PT and AG-3 TB in North Carolina was examined using somatic compatibility and amplified fragment length polymorphism (AFLP) criteria. A sample of 32 isolates from potato and 36 isolates from tobacco were paired in all possible combinations on PDA plus activated charcoal and examined for their resulting somatic interactions. Twenty-eight and eight distinct somatic compatibility groups (SCG) were identified in the AG-3 PT and AG-3 TB samples, respectively. AFLP analyses indicated that each of the 32 AG-3 PT isolates had a distinct AFLP phenotype, whereas 28 AFLP phenotypes were found among the 36 isolates of AG-3 TB. None of the AG-3 PT isolates were somatically compatible or shared a common AFLP phenotype with any AG-3 TB isolate. Clones (i.e., cases where two or
\end{abstract}

Accepted for publication October 26, 2001.

${ }^{1}$ Corresponding author, M.A. Cubeta; Email: marc_cubeta@ncsu. edu more isolates were somatically compatible and shared the same AFLP phenotype) were identified only in the AG-3 TB population. Four clones from tobacco represented $22 \%$ of the total population. All eight SCG from tobacco were associated with more than one AFLP phenotype. Compatible somatic interactions between AG-3 PT isolates occurred only between certain isolates from the same field (two isolates in each of four different fields), and when this occurred AFLP phenotypes were similar but not identical.

Key Words: clonality, genetic structure, mating, population structure, recombination

\section{INTRODUCTION}

Recent analysis of sequence variation in ribosomal DNA (rDNA) genes has identified at least two well defined phylogenetic groups of Rhizoctonia solani Kühn anastomosis group 3 (AG-3) that correspond to diseases associated with potato (AG-3 PT) and tobacco (AG-3 TB) (Kuninaga et al 1997, 2000, Gonzalez et al 2001). Although taxonomically related, the host range of isolates of $R$. solani AG-3 PT and AG-3 TB do not overlap, suggesting that AG-3 PT and AG3 TB may represent genetically subdivided populations among closely related species that have evolved a high level of specificity on different Solanaceous hosts (Ogoshi 1987, Stevens Johnk et al 1993, Shew and Melton 1995, Kuninaga et al 2000).

Rhizoctonia disease of potato (Solanum tuberosum L.) can occur on all plant parts. The fungus primarily infects underground portions of the plant and causes cankers on roots, sprouts, stems and stolons via mycelium and sclerotia that survive in soil and on seed potato tubers (Ogoshi 1987, Carling et al 1989). Unlike many Rhizoctonia diseases, this disease has an important seedborne (e.g., tuberborne) phase that provides a mechanism for long distance dispersal of the pathogen. Once established in soil, the mycelium and sclerotia of the pathogen may provide an additional source of primary inoculum.

In contrast to Rhizoctonia disease of potato, target spot disease of tobacco (Nicotiana tabacum L.) is not seedborne and occurs primarily on tobacco leaves. Target spot is initiated and spread via aerially dispersed meiospores (e.g., basidiospores) of Thanate- 


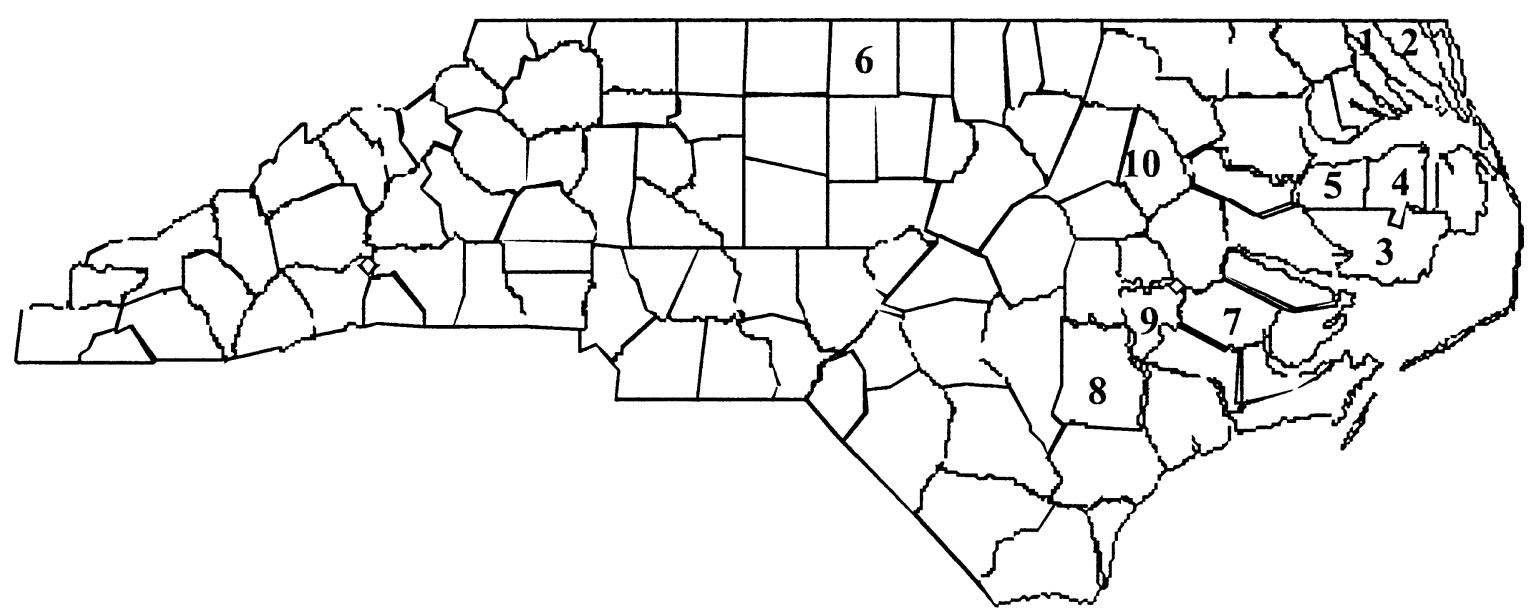

FIG. 1. Sampling locations for isolates of Rhizoctonia solani AG-3 PT and AG-3 TB in North Carolina counties. For AG-3 PT samples; 1 = Camden, 2 = Currituck, 3 = Hyde, 4 = Tyrrell and 5 = Washington. For AG-3 TB samples; $6=$ Caswell, $7=$ Craven, $8=$ Duplin, $9=$ Lenoir and $10=$ Edgecombe.

phorus cucumeris (Frank) Donk, the teleomorph of $R$. solani AG-3. Leaf infection occurs through direct penetration by hyphae from germinating basidiospores produced on hymenia on the soil or leaf surface. Basidiospores are an important source of primary and secondary inoculum in the tobacco pathosystem (Shew and Main 1985, Shew and Melton 1995), but there is no evidence that basidiospores play any role in the epidemiology of Rhizoctonia disease of potato.

Several previous studies have examined the genetic variation of $R$. solani AG-3 PT and AG-3 TB and revealed differences in their fatty acid and isozyme composition, rDNA genes and pathogenicity (Carling and Leiner 1990, Laroche et al 1992, Liu and Sinclair 1992, Liu et al 1993, Stevens Johnk et al 1993, Shew and Melton 1995, Kuninaga et al 1997, Ceresini et al 1999, Kuninaga et al 2000, Gonzalez et al 2001). Except for these studies, which employed a small sample of laboratory isolates, a comparison of the genetic diversity of individuals from field populations of $R$. solani AG-3 PT and AG-3 TB has not been conducted (Cubeta and Vilgalys 1997).

In this study, the hypothesis that populations of $R$. solani AG-3 TB are more genetically diverse than those from potato was tested based on a priori knowledge about the biology, ecology and epidemiology of each pathogen. To address this hypothesis, we used somatic compatibility and AFLP analyses to examine the genetic diversity and provide a foundation for examining the genetic structure of populations of $R$. solani AG-3 PT and TB in future experiments (Ceresini et al 2001).

\section{MATERIALS AND METHODS}

Isolates.-Thirty-two AG-3 PT and 36 AG-3 TB isolates of $R$. solani obtained from naturally infected plants from several commercial potato and tobacco production fields in North Carolina (NC) were examined in this study (FIG. 1, TABLES 1 AND 2). Six additional isolates of $R$. solani AG-3 PT (QSU02-8, PSB-03-8, dp216, L13, L71, and NK-04-7) representing a range of genetic diversity based on isozyme differences previously described by Laroche et al (1992) were also included. Isolates of $R$. solani AG-3 PT were collected from nine commercial potato production fields in five eastern NC counties in March 1997 (FIG. 1). Fourteen plots (two per field) with 6-8 rows of $9.14 \mathrm{~m}$ length each were planted with 320 seed pieces of potato (cv. 'Atlantic') previously disinfested with $2 \%$ formaldehyde to eliminate seedborne $R$. solani AG-3 PT (Carling et al 1989). All potato plants from each field plot were harvested and examined for stem canker symptoms. Samples were assayed on alkaline water agar (AWA, pH 8.5) to isolate $R$. solani AG-3 PT (Gutierrez et al 1997).

Isolates of AG-3 TB were obtained from 10 tobacco fields from five NC counties in June 1998 (FIG. 1). In each field, plants exhibiting target spot symptoms were collected from nine equally spaced locations within a $43 \times 55 \mathrm{~m}$ area. At each location within each field, five plants were sampled by taking one core (2.5-cm diameter) from each of five infected leaves. Leaf tissue samples were transferred to plates of AWA. After $24 \mathrm{~h}$ of incubation at room temperature, hyphal tips of colonies with growth characteristics of $R$. solani were transferred to new AWA and then to potato dextrose agar (PDA) to establish axenic cultures. Isolates were identified and maintained on sterilized rice grains at -20 C. Nuclear condition of isolates was determined in cultures grown on AWA on glass slides by the DAPI staining protocol (Martin 1987). The anastomosis group of all isolates was determined on glass slides containing a thin layer of AWA pH 8.5 (Herr and Roberts 1980) against the AG-3 tester isolate SCL24. The reaction was considered positive when at least five pairs of hyphae exhibited the C2 anastomosis reaction on each of four slides (Carling 1996).

Determination of somatic compatibility grouping.-To determine somatic compatibility, AG-3 PT and AG-3 TB isolates 
were paired in all possible combinations on potato dextrose agar (3\% Difco PDA) amended with 1\% charcoal (Butler and Bolkan 1973) according to the procedure of MacNish et al (1997). The isolates were grown for 4 days at $25 \mathrm{C}$ and somatic interactions between isolates were determined macroscopically. Pairings were scored as somatically compatible when no reaction line was observed between paired isolates. Pairings were scored as somatically incompatible when a reaction line of raised hyphae was observed between paired isolates (MacNish et al 1997). Somatic compatibility experiments were conducted at least twice. Anastomosis reactions between pairs of representative isolates from each host were determined microscopically on glass slides containing a thin layer of AWA pH 8.5 (Herr and Roberts 1980). The categories of hyphal anastomosis reactions were examined according to Carling (1996) as follows: C2, indicates same anastomosis group but different somatic compatibility group (SCG); and C3, indicates closely related isolates, same anastomosis group, same SCG, or same individual. A phenogram was constructed using neighbor-joining phenotypic clustering (Saitou and Nei 1987) performed on the matrix of Manhattan phenotypic distances (Nei 1987) to examine the relationships among AG-3 TB isolates. The analysis was applied to binomially coded data for each reaction phenotype, compatible (1) or incompatible (0).

DNA extraction.-Total genomic DNA was extracted from lyophilized mycelium of $R$. solani AG-3 grown for $5 \mathrm{~d}$ at 25 $\mathrm{C}$ in potato-dextrose broth (Difco). DNA for AFLP analysis was prepared by extraction with SDS buffer and phenolchloroform as previously described by Vilgalys and Gonzalez (1990).

AFLP analysis.-The AFLP analysis procedure was a modification of Vos et al (1995). Approximately $150 \mathrm{ng}$ of genomic DNA was digested for at least $2.5 \mathrm{~h}$ at $37 \mathrm{C}$ with $5 \mathrm{U}$ of Eco RI (Promega) and $5 \mathrm{U}$ of Mse I (Gibco BRL). The $40 \mu \mathrm{L}$ reaction volumes included the following components: $10.4 \mathrm{mM}$ Tris $\mathrm{HCl}(\mathrm{pH} 7.4), 10 \mathrm{mM} \mathrm{Mg}$ acetate, 50 $\mathrm{mM} \mathrm{K}$ acetate, $5.4 \mathrm{mM} \mathrm{NaCl}, 0.004 \mathrm{mM}$ EDTA, $5.035 \mathrm{mM}$ dithiothreitol, $0.06 \mathrm{mg} / \mathrm{mL}$ bovine serum albumin, $1.77 \%$ (v/v) glycerol, $0.004 \%$ Triton X-100. The templates for PCR amplification were generated by ligating the Mse I-double stranded (ds) adapter (MA1 and MA2) and Eco RI-ds adapter sequences (EA1 and EA2) to the Mse I (MA1, 5'-GACGATGAGTCCTGAG-3' + MA2, 3'-TACTCAGGACTCAT-5') and Eco RI sites (EA1, 5'-CTCGTAGACTGCGTACC-3' + EA2, 3'-CATCTGACGCATGGTTAA-5') of the double digested fragments of DNA using 1 Weiss unit of T4 ligase (Promega) per $50 \mu \mathrm{L}$ reaction. After ligation, the reaction mixture was diluted ten-fold with sterile deionized water and stored at $-20 \mathrm{C}$. The first amplification employed Mse $\mathrm{I}$ and Eco RI primers with one selective nucleotide. Two pairs of primers were used: Eco RI primer $+\mathrm{C}\left(5^{\prime}-\right.$ GACTGCGTACCAATTC $\left.C-3^{\prime}\right)$ and Mse I primer + A $\left(5^{\prime}-\right.$ GATGAGTCCTGAGTAAA-3'); Eco RI primer + G (5'GACTGCGTACCAATTC $\left.G 3^{\prime}\right)$ and Mse I primer + G $\left(5^{\prime}-\right.$ GATGAGTCCTGAGTAA $\left.G 3^{\prime}\right)$. The sample included $5 \mu \mathrm{L}$ of DNA template, $1 \mu \mathrm{M}$ of Mse I primer, $1 \mu \mathrm{M}$ of Eco RI primer, $0.2 \mathrm{mM}$ dNTPs, $1 \mathrm{mM} \mathrm{MgCl}{ }_{2}$, enzyme-specific PCR buffer and 2.5 U of AmpliTaq DNA polymerase, Stoffel frag- ment (Perkin-Elmer). The PCR was performed in a thermal cycler (Perkin-Elmer) for 20 cycles of $30 \mathrm{~s}$ at $94 \mathrm{C}, 1 \mathrm{~min}$ at $56 \mathrm{C}$ and $1 \mathrm{~min}$ at $72 \mathrm{C}$.

For selective amplification of the secondary template, Eco RI and Mse I primers with two selective nucleotides were used. Two pairs of selective primers with the following sequences were used: Eco RI primer + CA (5'-GACTGCGTACCAATTC $\left.C A-3^{\prime}\right)$ and $M s e$ I primer + AC (5'-GATGAGTCCTGAGTAA $\left.A C-3^{\prime}\right)$; Eco RI primer + GT $\left(5^{\prime}-\right.$ GACTGCGTACCAATTCGT-3') and Mse I primer + GT (5'GATGAGTCCTGAGTAAGT-3'). The Eco RI primer + CA was end-labeled with fluorescent dye 9-TET and the Eco RI primer + GT with fluorescent dye 6-FAM, at the $5^{\prime}$ end. All primers were synthesized by Genosys Biotechnologies, Inc.

The selective PCR mixture included, for each $25 \mu \mathrm{L}$ of reaction, $5 \mu \mathrm{L}$ of secondary DNA template obtained from non-selective amplification, $1 \mu \mathrm{M}$ of the Mse I selective primer, $1 \mu \mathrm{M}$ of the Eco RI selective primer, $0.2 \mathrm{mM}$ dNTPs, $1 \mathrm{mM} \mathrm{MgCl}_{2}$, enzyme-specific PCR buffer and $2.5 \mathrm{U}$ of AmpliTaq DNA polymerase, Stoffel fragment (Perkin-Elmer).

Analysis of AFLP data.-Following PCR amplification, products were diluted 10 -fold with sterile distilled $\mathrm{H}_{2} \mathrm{O}$. A volume of $1-2 \mu \mathrm{L}$ of the resulting diluted samples was vacuumdried, mixed with the fluorescent sequencing dye TAMRA500 containing internal molecular marker standard (Genset Oligos) at $2 \times$ concentration, denatured at $90 \mathrm{C}$ for $5 \mathrm{~min}$ and placed in ice. A $1 \mu \mathrm{L}$ sample of each reaction was loaded on a $4.5 \%$ denaturing polyacrylamide gel, in a ABI model 377 sequencing electrophoresis system (Applied Biosystems). The running buffer was $1 \times \mathrm{TBE}$ and electrophoresis was performed at $80 \mathrm{~W}$ for $2.5 \mathrm{~h}$. The electrophoresis gel files generated by this system were handled with the objective of extracting raw information contained in each gel column with the software GeneScan (ABIPRISM, Applied Biosystems). This software automatically analyzes the information collected by the sequencer; it identifies, quantifies, and determines the sizes of DNA fragments. The AFLP profiles were scored with the software Genographer (Montana State University). Because of the difficulty in inferring common descent from common state when comparing co-migrating fragments, each AFLP pattern was treated as a phenotype. The AFLP phenotypes for potato and tobacco isolates were obtained based on the scoring of 63 polymorphic bands (27 generated from CA-AC and from GT-GT primer combination), binomially coded as present (1) or absent (0). The reproducibility of the AFLP analysis was controlled by scoring fragments against the AFLP profile from two isolates of $R$. solani AG-3 (9/P217 and 11/T032) from two different DNA extractions, included in all gels.

Data analyses.-Each isolate of $R$. solani AG-3 was characterized based on its somatic compatibility grouping and AFLP phenotype. Clones were defined as a group of isolates that share a unique AFLP phenotype and belong to the same SCG. AFLP phenotypes were scored by presence and/ or absence of shared bands. A phenogram was generated to group isolates based on their similarity according to their AFLP profile. Neighbor-joining phenotypic clustering (Saitou and Nei 1987) was performed on the matrix of Man- 


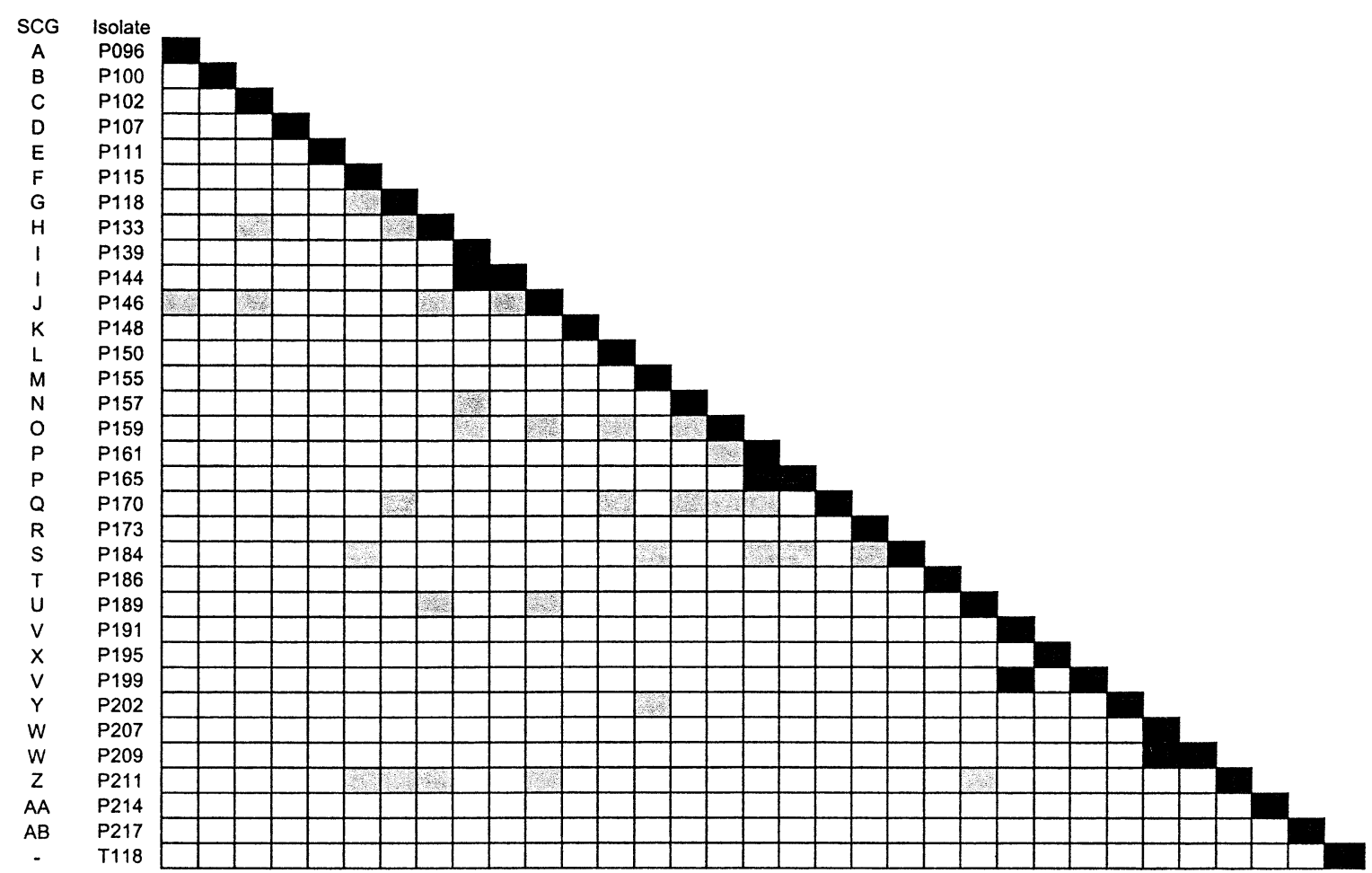

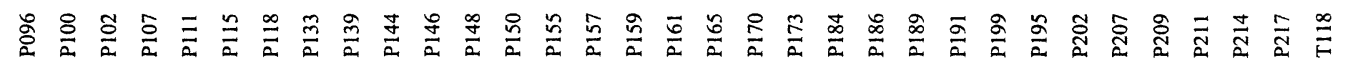

FIG. 2. Macroscopic somatic interactions among potato stem canker isolates of Rhizoctonia solani AG-3 PT on potato dextrose agar amended with $1 \%$ charcoal. Isolates for each pairing were grown for 4 days at $25 \mathrm{C}$ and examined for their somatic interactions. The categories of macroscopic somatic interactions were: $\boldsymbol{\square}=$ somatically compatible, where paired isolates grew together without a distinct demarcation; $\square=$ somatically incompatible, represented by distinct demarcation between cultures and raised hyphae in the interaction zone; and $=$ weak incompatible reaction (MacNish et al 1997). T118 is an isolate from AG-3 TB that was somatically incompatible with all AG-3 PT isolates.

hattan phenotypic distances (Nei 1987) with the software PHYLIP 3.5 (Felsenstein 1995).

\section{RESULTS}

Determination of somatic compatibility grouping.-Four pairs of AG-3 PT isolates (from a sample of 32) were somatically compatible: 5/P139 and 5/P144, 6/P161 and $6 / \mathrm{P} 165,8 / \mathrm{P} 191$ and 8/P199, and 9/P207 and 9/P209 (FIG. 2). Isolates from each of these compatible pairs were collected from infected potato stems in same field. The six additional isolates of AG-3 from potato (QSU-02-8, PSB-03-8, dp216, L13, L71, and NK-04-7), representing different groups of isozyme variation previously reported within AG-3 PT (Laroche et al 1992) were somatically incompatible with one another and all NC potato isolates (data not shown). Eight SCG were identified in the AG-3 TB sample (FIGS. 3 AND 4). The phenotypic clustering of AG-3 TB isolates of $R$. solani AG-3 according to SCG is presented in the FIG. 4. In the AG-3 TB sample, there were two cases of non-transitivity (i.e., where one isolate of a distinct SCG was compatible with an- other isolate belonging to a different SCG). Nontransitivity was observed between 10 pairs of isolates: 10/T007 (SCG B) and 12/T051 (SCG D), 12/T039 (SCG B) and 19/T149 (SCG A), 10/T001 (SCG D) and 14/T068 (SCG C), 11/T029 (SCG F) and 17/ T118 (SCG E), 11/T029 (SCG F) and 18/T147 (SCG E), 19/T160 (SCG H) and 12/T043 (SCG G), 19/ T160 (SCG H) and 15/T095 (SCG G), 13/T052 (SCG H) and 15/T095 (SCG G), 13/T052 (SCG H) and 19/T152 (SCG G), 16/T100 (SCG E) and 19/ T160 (SCG H) (Fig. 3). However, there was no intransitivity between any of the isolates from SCG A, B, C and D with any members of SCG E, F, G and H. One incompatible reaction (12/T035 and 11/T032) within a compatible group (SCG D) was observed. Weakly incompatible reactions between isolates of $R$. solani AG-3 were observed in AG-3 PT (3.1\% of total pairings) and AG-3 TB samples $(9.8 \%$ of total pairings, gray-colored boxes in the FIGs. 2 AND 3). No examples of somatic compatibility were observed between AG-3 PT and AG-3 TB isolates.

AFLP analysis.-From 105 AFLP fragments scored with two different sets of primers (Eco RI-CA / Mse 


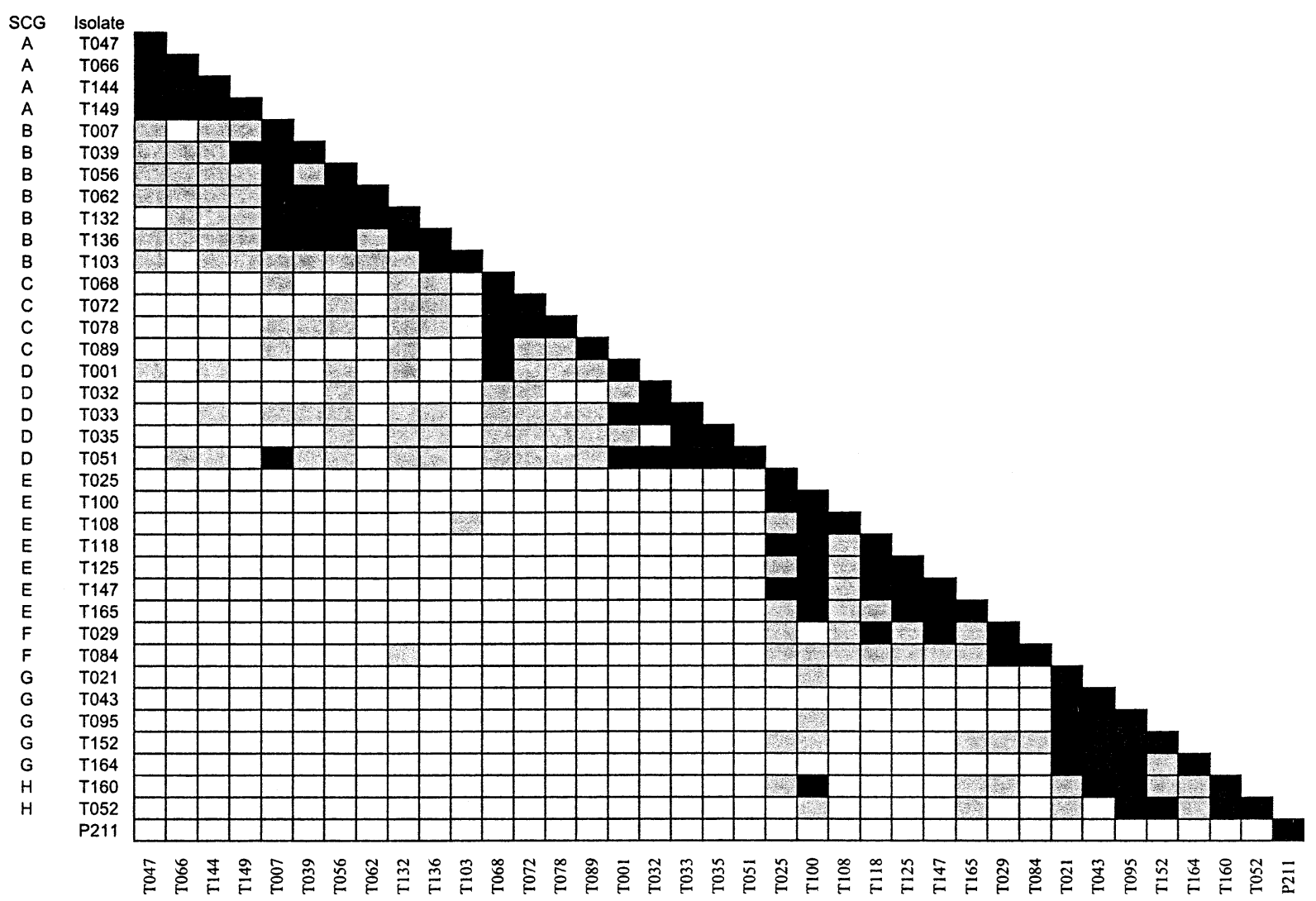

FIG. 3. Macroscopic somatic interactions among Rhizoctonia solani AG-3 TB isolates on potato dextrose agar amended with $1 \%$ charcoal. Isolates for each pairing were grown for $4 \mathrm{~d}$ at $25 \mathrm{C}$ and examined for their somatic interactions. The categories of macroscopic somatic interactions were: $\mathbf{\square}=$ somatically compatible, where paired isolates grew together without a distinct demarcation; $\square=$ somatically incompatible, represented by distinct demarcation between cultures and raised hyphae in the interaction zone; and = weak incompatible reaction (MacNish et al 1997). P211 is an isolate from AG-3 PT that was somatically incompatible with all AG-3 TB isolates.

I-AC and Eco RI-GT / Mse I-GT), $45 \%$ were polymorphic and $1 \%$ were monomorphic among AG-3 PT isolates, and 54\% were detected only in AG-3 TB isolates. In contrast, $15 \%$ of the bands were polymorphic and $43 \%$ were monomorphic among AG-3 TB isolates, and $42 \%$ were detected only on AG-3 PT isolates. The AFLP phenotypes for AG-3 PT and AG-3 TB isolates were obtained based on the scoring of 63 polymorphic bands.

Thirty-two AFLP phenotypes were identified among isolates of $R$. solani AG-3 PT (FIG. 5, TABLE I) and 28 among isolates of AG-3 TB (FIG. 4, TABLE II). There were no common AFLP patterns shared among isolates of $R$. solani AG-3 PT and AG-3 TB (TABLES I AND II). The additional isolates of AG-3 PT (QSU-02-8, PSB-03-8, dp216, L13, L71, and NK-047) included in this study also had distinct AFLP phenotypes (TABLES I AND II).

There were no clones identified among isolates of AG-3 PT (i.e., cases in which two or more isolates belonged to the same SCG and shared the same
AFLP phenotype). Among AG-3 TB isolates, four clones were identified: 18/T147 and 16/T108 (SCG E); 17/T118 and 17/T125 (SCG E); 14/T068 and 14/T072 (SCG C); 12/T047 and 13/T066 (SCG A). These clones, represented $22 \%$ of the AG-3 TB isolates. There were two cases among the tobacco sample in which one AFLP phenotype was associated with more than one SCG, as observed with isolates 18/ T136/B, 18/T147/E or 16/T108/E (with fingerprint designation \#1), and19/T-152/G, 13/T052/H or 18/ T132/B, with fingerprint designation \#4 (FIG. 4, TABLES II AND III). All eight SCG of AG-3 TB isolates were associated with more than one AFLP phenotype (Fig. 5, TABles II AND III).

Isolates of $R$. solani AG-3 TB with different SCG and AFLP patterns were recovered from each individual tobacco field from most of the counties sampled (TABLE III). For example, from a field in Caswell County, one isolate of SCG A, one of SCG E, two of SCG $\mathrm{G}$ and one from SCG $\mathrm{H}$ were recovered. Fields in Craven and Edgecombe Counties, had a single 


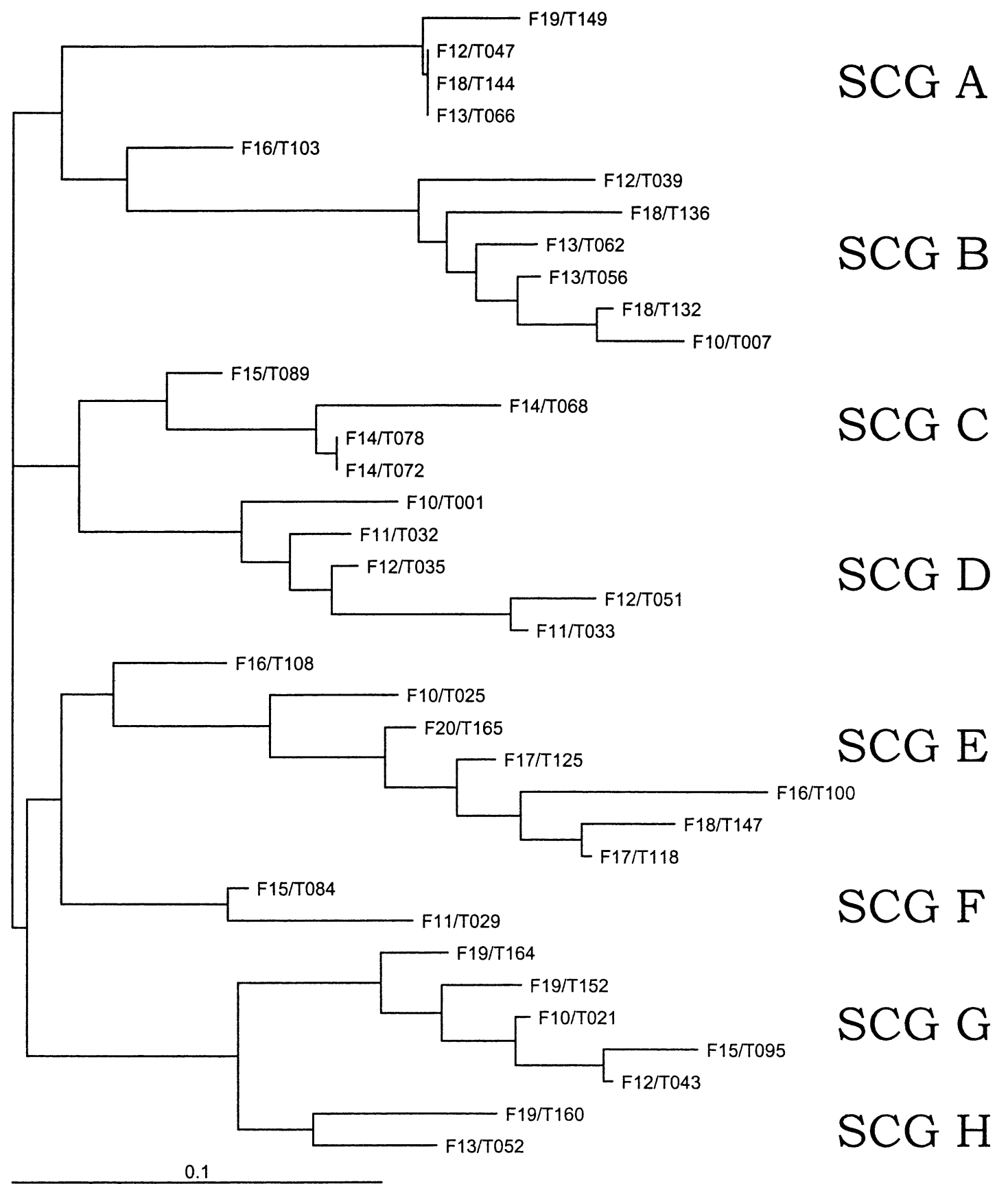

FIG. 4. Phenogram of somatic compatibility grouping (SCG) of Rhizoctonia solani AG-3 TB isolates generated by neighborjoining method (Saito and Nei 1987) performed on the matrix of Manhattan phenotypic distances (Nei 1987) with the software PHYLIP 3.5 (Felsenstein 1995).

SCG present. In terms of geographical distribution of clones (TABLE III), two clones were recovered from more than one field in the same (12/T047/A and 13/T066/A from Craven) or different counties in NC (16/T108/E and 18/T147/E from Edgecombe and Caswell, respectively) (TABLE III). Edgecombe and Caswell Counties are located approximately 220 $\mathrm{km}$ apart.

\section{DISCUSSION}

The primary objective of this study was to examine the genetic diversity of field populations of $R$. solani AG-3 PT and AG-3 TB in NC and to provide a foundation for understanding the distribution of this genetic diversity on a spatial scale for future experiments (Ceresini et al 2001). Based on a priori knowledge about the biology, ecology and epidemiology of 


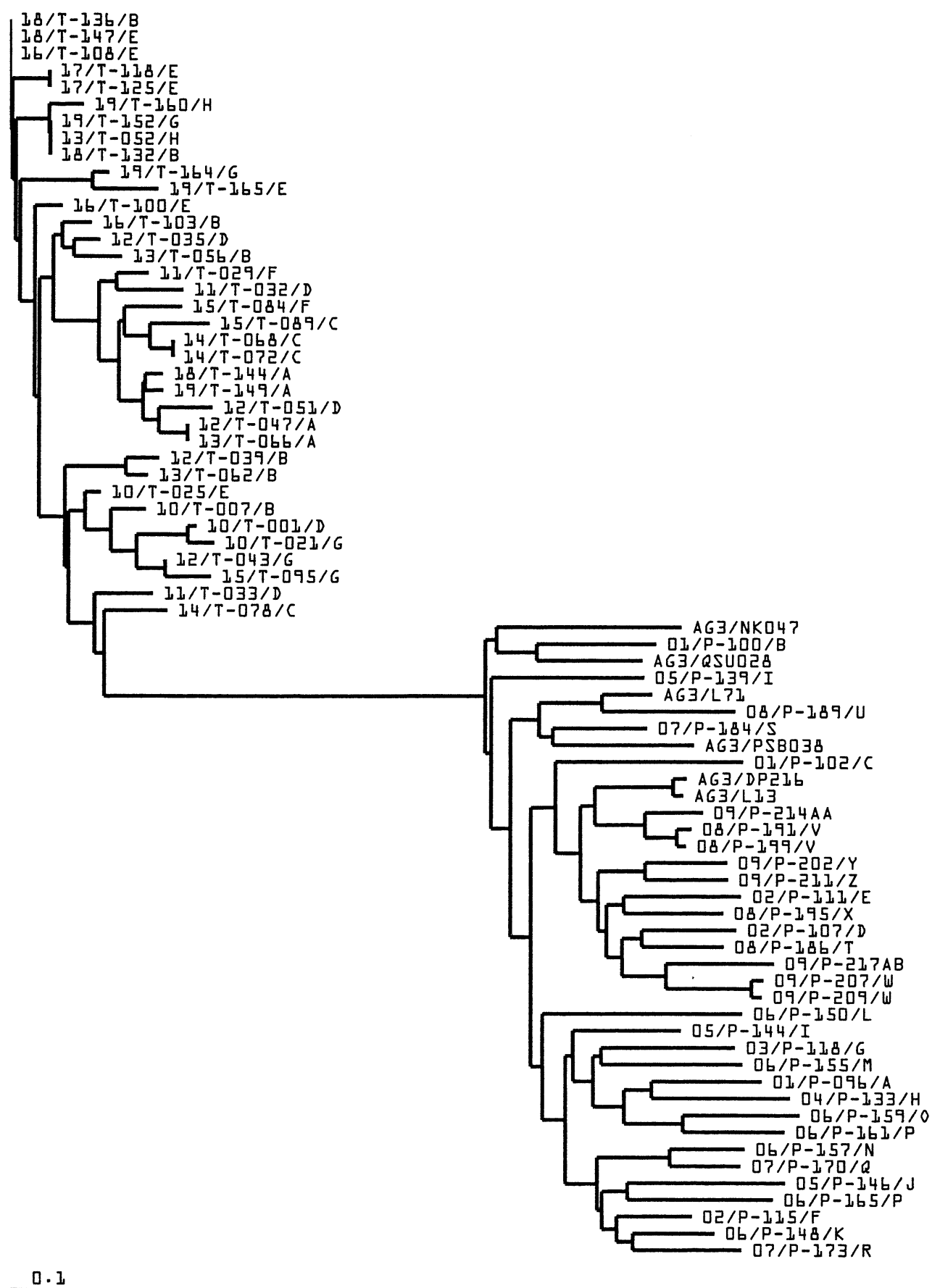

FIG. 5. Phenogram of DNA fingerprinting based on AFLP phenotypes of Rhizoctonia solani AG-3 PT and AG-3 TB isolates generated by neighbor-joining method (Saito and Nei 1987) performed on the matrix of Manhattan phenotypic distances (Nei 1987) with the software PHYLIP 3.5 (Felsenstein 1995). The designation of each isolate represents its field of origin, the isolate code and its somatic compatibility group (SCG). For example, the isolate $07 / \mathrm{P}-173 / \mathrm{R}$ is from the potato field 7 , code P-173 and belongs to SCG R. Additional information is presented in the TABLES I AND II.

R. solani AG-3, we hypothesized that field isolates of tobacco would harbor greater genetic diversity than potato isolates.

Based on two independent criteria, our results indicated that isolates of AG-3 TB are represented by fewer SCG and AFLP phenotypes than isolates of AG-
3 PT. In addition, four clones representing 22\% of the AG-3 TB isolates sample were detected, whereas no clones were detected among isolates of AG-3 PT from NC. Two clones of AG-3 TB also were recovered from more than one field in the same or in widely separated counties across the area of tobacco pro- 

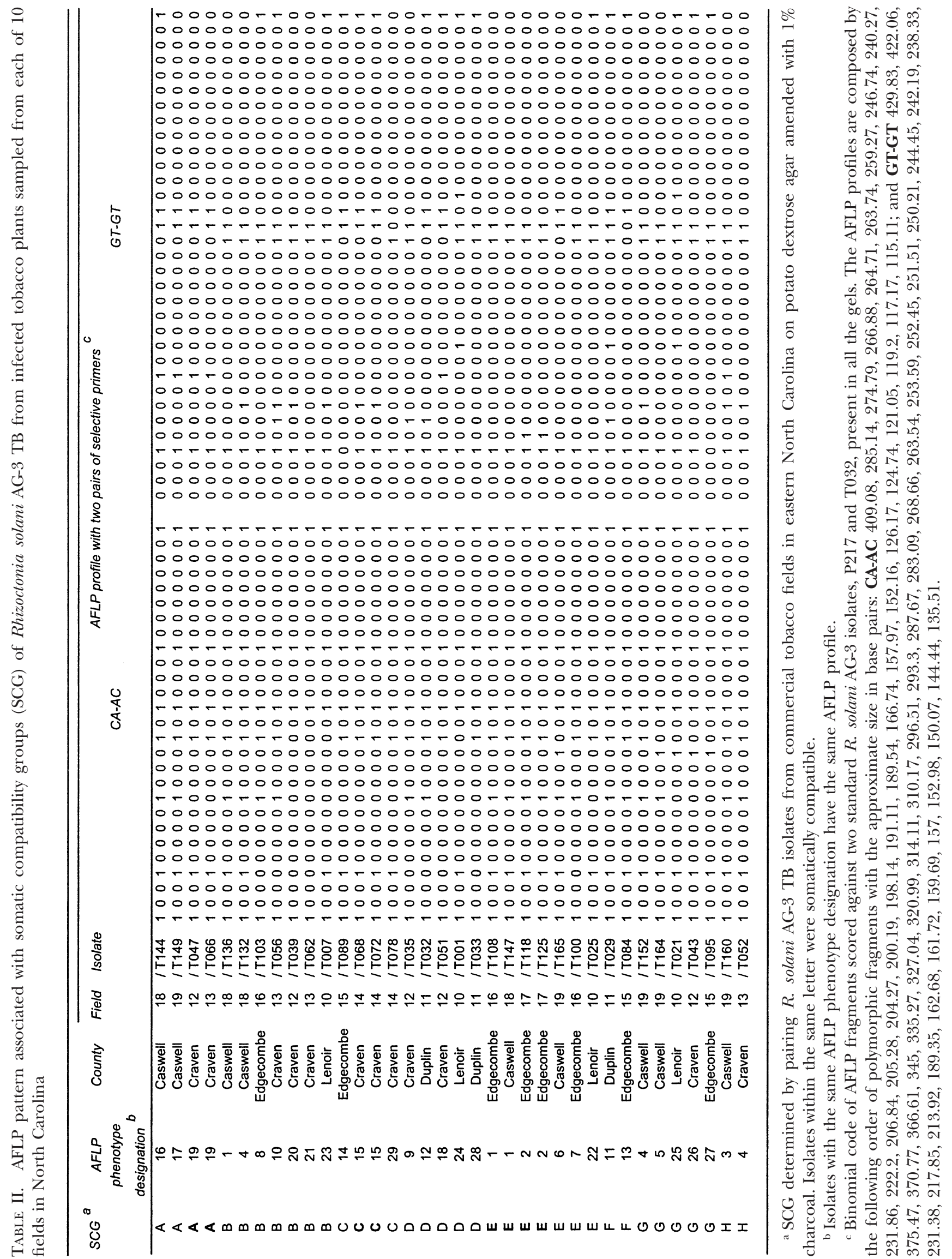


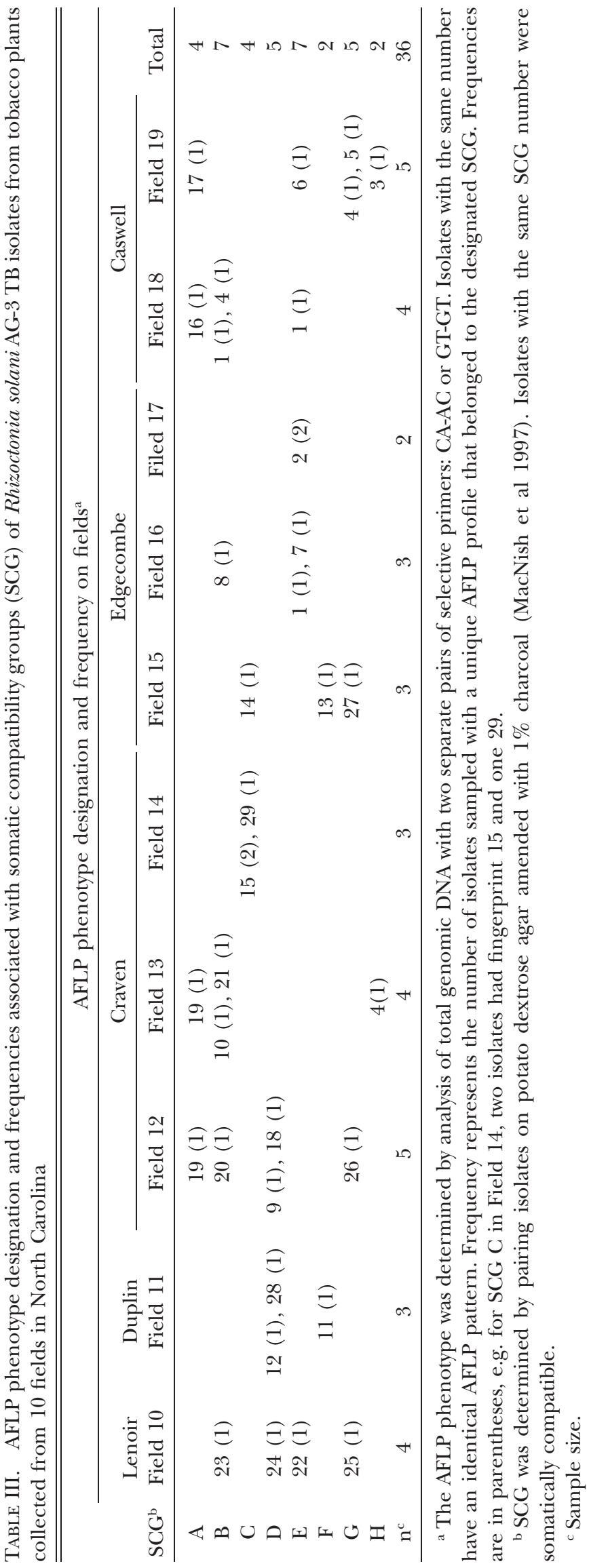

duction in NC. Therefore, the hypothesis that populations of $R$. solani AG-3 TB are more genetically diverse than those of AG-3 PT was rejected. The lack of common AFLP phenotypes and compatible somatic interactions observed among potato and tobacco isolates supports that populations of $R$. solani AG3 are subdivided by host specialization (Kuninaga et. al 1997, Ogoshi 1987, Stevens Johnk et al 1993, Gonzalez et al 2001).

Transportation of infested potato seed tubers from northern production areas in the US and Canada to NC plays an important role in the geographic distribution of AG-3 PT and possibly contributed to the high level of genetic diversity observed among isolates in this study. In contrast to the potato-AG-3 PT pathosystem, the distribution and occurrence of the AG-3 TB in commercial production fields may result from airborne movement of basidiospores of T. cucumeris. While long distance transport (approximately $220 \mathrm{~km}$ between Edgecombe and Caswell Counties) may be possible, the hyaline, thin-walled basidiospores of T. cucumeris suggest that long distance dispersal is unlikely. An alternative explanation could be the movement of sclerotia and mycelium in soil associated with local tobacco transplant production in NC.

The utilization of independent criteria, such as somatic incompatibility and AFLP analysis, can provide preliminary information about mating systems and population structure. For example, progeny generated via asexual or homothallic sexual (self-fertile, inbreeding) reproduction will be genetically identical (or nearly identical) to the parental isolate and all parts of the genome will have the same evolutionary history because of the lack of recombination (Worrall 1997, Taylor et al 1999). Depending on the age and size of the population, no or limited recombination would contribute to the association between independent characters (genetic markers), a population with limited genetic diversity, and the repeated recovery of similar genotypes (e.g., clonal population structure). In contrast, progeny generated via heterothallic sexual (outcrossing) reproduction will be genetically different than parental isolates as a result of recombination of genetically different nuclei. Therefore, different regions of the genome of the progeny will have different evolutionary histories (Taylor et al 1999). In this case, recombination reduces (or eliminates) the one-to-one (strict) association between independent characters and results in a non-clonal population structure (Anderson and Kohn 1995, Milgroom 1996, Cubeta et al 1997).

Although our sample size was insufficient to make any inferences concerning the contribution of the observed genetic diversity to population structure in 
this study, several relationships between somatic compatibility and AFLP data were evident. For example, the AG-3 TB population appeared to have a clonal component based on repeated recovery of clones from different counties; however, there was also evidence for recombination. Among the AG-3 TB population, there were deviations from the strict association between SCG and AFLP. In general, the eight SCG from tobacco were associated with at least two AFLP phenotypes. However, SCG B was associated with seven, SCG E with six, and SCG D and G with five different AFLP phenotypes. There were also two cases among the tobacco isolates in which one AFLP phenotype was associated with more than one SCG. There were also examples of non-transitivity in somatic compatibility among isolates of AG-3 TB. For example, isolate 12/T051 was compatible with isolate 10/T001; isolate 12/T051 was compatible with 10/ T007, but isolate 10/T001 was incompatible with 10/ T007. Repeated examination of these pairings in separate experiments indicated consistent observation of non-transitivity. Results similar to those observed in this study with non-transitivity have also been observed for Sclerotinia sclerotiorum, and for the Basidiomycetes Suillus granulatus and Pleurotus ostreatus (Jacobson et al 1993, Cubeta et al 1997, Malik and Vilgalys 1999).

Isolates from the same SCG and with the same AFLP phenotype were compatible (FIG. 6). There were several cases of weak incompatible reactions observed among the pairings within the AG-3 PT or AG3 TB isolates. These reactions were characterized by a small raised area in the hyphal interaction zone (FIG. 7). However, among some groups of isolates with the same AFLP phenotype in AG-3 TB, a range of incompatibility reactions was observed that varied in intensity (Figs. 7 AND 8). Factors such as the genetic relatedness of the interacting isolates, the age of the mycelium and the pairing distance may account for the intensity of the observed macroscopic hyphal interactions. However, the biological significance of these weak macroscopic incompatible reactions is not known.

The range of somatic interactions among pairings of AG-3 TB and deviations from a strict association of SCG and AFLP are consistent with recombination. In $S$. granulatus, somatic mutation or recombination was considered to be involved in some of the variation observed with RAPD markers (Jacobson et al 1993). The observed non-transitivity could be related to allelic differences at the somatic incompatibility loci that are necessary for detecting somatic incompatibility (Malik and Vilgalys 1999).

The genetic regulation of somatic incompatibility in T. cucumeris is not known. However, our observa-
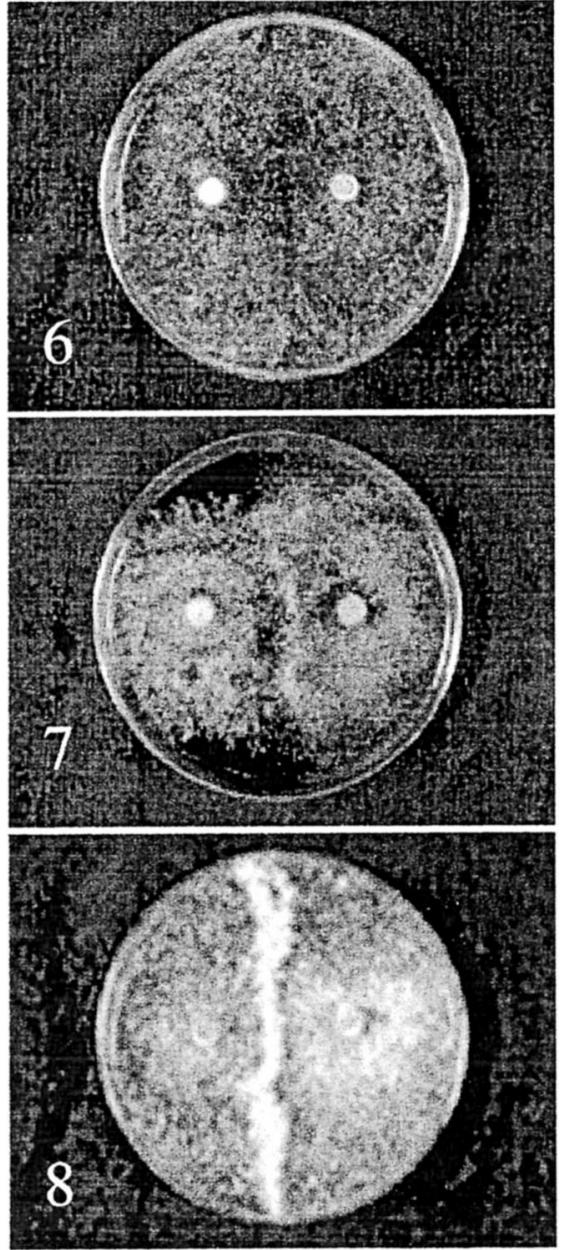

FIGS. 6-8. Macroscopic somatic interactions among potato stem canker isolates of Rhizoctonia solani AG-3 PT on potato dextrose agar amended with $1 \%$ charcoal. 6 . Somatically compatible interaction (no distinct demarcation in the hyphal interaction zone between paired isolates); 7 . Weak incompatible interaction (demarcation between paired isolates in the hyphal interaction zone and manifested as small areas of raised hyphae); 8. Somatically incompatible interaction (demarcation between paired isolates in the hyphal interaction zone and manifested as large areas of raised hyphae).

tions are consistent with somatic incompatibility mediated by the sharing of some, but not all, compatibility loci or alleles. In the few cases for which there is evidence, somatic compatibility in basidiomycetes appears to be controlled primarily by nuclear genes that are not linked with mating compatibility loci (Kay and Vilgalys 1992, Hansen et al 1993, Rizzo et al 1995). Because of the frequency of somatic incompatibility among field isolates and closely related heterokaryons, multiple loci and/or multiple alleles are thought to control somatic compatibility. Differences at one locus would lead to somatic incompatibility, but in most cases somatic incompatibility is more in- 
tense with greater genetic differences (Hansen et al 1993, Malik and Vilgalys 1999).

This study provides some preliminary information about the population structure of $R$. solani AG-3 PT and TB. Although the sampling strategies employed in this study were probably sufficient for maximum detection of genetic diversity, additional sampling within larger fields is needed to elucidate the magnitude of clonality and/or recombination in local populations of this pathogen. The ability to survey a large number of loci with AFLP analysis provides a useful method for identification of genetically distinct individuals in populations with a high level of resolution. However, AFLP are dominant genetic markers, a characteristic that limits their potential utility in classical population genetics analyses. Therefore, our laboratory has focused on the development of co-dominant markers (e.g., restriction fragment length polymorphism $=$ RFLP) for multilocus genotyping of $R$. solani AG-3 isolates (Ceresini et al 2001). Co-dominant markers enable unambiguous scoring of genotypes of $R$. solani AG-3 and provide a means of distinguishing between homozygous and heterozygous individuals from a population. These markers are more appropriate for elucidating the evolutionary processes that shape populations of $R$. solani AG3. Successful use of co-dominant RFLP markers for population genetic studies of $R$. solani has been previously demonstrated for the AG-1-IA pathosystem on rice (Rosewich et al 1999).

\section{ACKNOWLEDGMENTS}

For helping with the sampling of potato and tobacco isolates of $R$. solani we thank Bryan Cody and Walter Gutierrez. For his patience and use of laboratory space, we thank Steve Leath (USDA/ARS, Dept. of Plant Pathology, NCSU). Thanks to Anja Forche and Shian-ren Liou, and Daniel Snyder (Duke University, Dept. of Biology) for the advice with AFLP data collection and analysis; and Pamela Puryear (Tobacco Literature Service, NCSU) for literature searches. This research was supported in part with a research assistantship from $\mathrm{CNPq}$ (Conselho Nacional de Desenvolvimento Científico e Tecnológico)—Brasília, DF, Brazil (200873/92-8). A leave of absence was also granted to PC by UNESP (Universidade Estadual Paulista "Júlio de Mesquita Filho"), Ilha Solteira, São Paulo, Brazil, to attend a Ph.D. program in Plant Pathology at North Carolina State University. Special thanks to Drs. Ana Maria R. Cassiolato and Marli F. Stradioto Papa for assuming the teaching responsibilities of PC at UNESP during his absence.

\section{LITERATURE CITED}

Anderson JB, Kohn LM. 1995. Clonality in soilborne plantpathogenic fungi. Annu Rev Phytopathology 33:369391.
Butler EE, Bolkan H. 1973. A medium for heterokaryon formation in Rhizoctonia solani. Phytopathology 63: 542-543.

Carling DE, Leiner RH, Westphale PC. 1989. Symptoms, signs, and yield reduction associated with rhizoctonia disease of potato induced by tuberborne inoculum of Rhizoctonia solani AG-3. Am Potato J 66:693-702.

- - 1990. Virulence of isolates of Rhizoctonia solani AG-3 collected from potato plant organs and soil. Plant Dis 74:901-903.

- 1996. Grouping in Rhizoctonia solani by hyphal anastomosis reaction. In: Sneh B, Jabaji-Hare S, Neate S, Dijst G, eds. Rhizoctonia species: taxonomy, molecular biology, ecology, pathology and disease control. Dordrecht, The Netherlands: Kluwer Academic Publishers. p 37-47.

Ceresini PC, Shew HD, Cubeta MA. 1999. RFLP analysis of the PCR amplified ribosomal DNA regions ITS and IGS indicated that isolates of Rhizoctonia solani from potato and tobacco represent distinct groups within the anastomosis group 3. Phytopathology 89:S12.

—, Vilgalys R, Rosewich UL, Cubeta MA. 2001. Genetic structure of populations of Rhizoctonia solani AG-3 from potato in eastern North Carolina. Mycologia 93:(Submitted companion paper)

Cubeta MA, Vilgalys R. 1997. Population biology of the Rhizoctonia solani complex. Phytopathology 87:480-484.

—, Cody BR, Kohli Y, Kohn LM. 1997. Clonality of Sclerotinia sclerotiorum from cabbage in eastern North Carolina. Phytopathology 87:1000-1004.

Felsenstein J. 1995. PHYLIP (Phylogeny Inference Package) v. 3.57c. Seattle, Washington: Department of Genetics, The University of Washington.

Gonzalez D, Carling DE, Kuninaga S, Vilgalys R, Cubeta MA. 2001. Ribosomal DNA systematics of Ceratobasidium and Thanatephorus with Rhizoctonia anamorphs. Mycologia 93:1138-1150.

Gutierrez WA, Shew HD, Melton TA. 1997. Sources of inoculum and management for Rhizoctonia solani damping-off on tobacco transplants under greenhouse conditions. Plant Dis 81:604-606.

Hansen EM, Stenlid J, Johansson M. 1993. Genetic control of somatic incompatibility in the root-rotting basidiomycete Heterobasidion annosum. Mycol Res 97:12291233.

Herr LJ, Roberts DL. 1980. Characterization of Rhizoctonia solani populations obtained from sugar beet fields with differing soil textures. Phytopathology 70:476-480.

Jacobson KM, Miller OK Jr, Turner BJ. 1993. Randomly amplified polymorphic DNA markers are superior to somatic incompatibility tests for discriminating genotypes in natural populations of the ectomycorrhizal fungus Suillus granulatus. Proc Natl Acad Sci USA 90:91599163.

Kay E, Vilgalys R. 1992. Spatial distribution and genetic relationship among individuals in a natural population of the oyster mushroom Pleurotus ostreatus. Mycologia 84:173-182.

Kuninaga S, Natsuaki T, Takeuchi T, Yokosawa R. 1997. Sequence variation of the rDNA ITS regions within and 
between anastomosis groups in Rhizoctonia solani. Curr Genet 32:237-243.

- Carling DE, Takeuchi T, Yokosawa R. 2000. Comparison of rDNA ITS sequences between potato and tobacco strains in Rhizoctonia solani AG-3. J Gen Plant Pathol 66:2-11.

Laroche JP, Jabaji-Hare SH, Charest PM. 1992. Differentiation of two anastomosis groups of Rhizoctonia solani by isozyme analysis. Phytopathology 82:1387-1393.

Liu ZL, Sinclair JB. 1992. Genetic diversity of Rhizoctonia solani anastomosis group 2. Phytopathology 82:778787.

-, Domier LL, Sinclair JB. 1993. ISG-specific ribosomal DNA polymorphism of the Rhizoctonia solani species complex. Mycologia 85:795-800.

MacNish GC, Carling DE, Brainard KA. 1997. Relationship of microscopic and macroscopic vegetative reactions in Rhizoctonia solani and the occurrence of vegetatively compatible populations (VCPs) in AG-8. Mycol Res 101:61-68.

Malik M, Vilgalys R. 1999. Somatic incompatibility in fungi. In: Worrall JJ, ed. Structure and Dynamics of Fungal Populations. Dordrecht, The Netherlands: Kluwer Academic Publishers. p 123-138.

Martin B. 1987. Rapid tentative identification of Rhizoctonia spp. associated with diseased turfgrasses. Plant Dis 71: 47-49.

Milgroom MG. 1996. Recombination and the multilocus structure of fungal populations. Annu Rev Phytopathol 34:457-477.

Nei M. 1987. Molecular Evolutionary Genetics. New York: Columbia University Press. 512 p.
Ogoshi A. 1987. Ecology and pathogenicity of anastomosis and intraspecific groups of Rhizoctonia solani Kühn. Annu Rev Phytopathol 25:125-143.

Rizzo DM, Rentmeester RM, Burdsall HH Jr. 1995. Sexuality and somatic incompatibility in Phellinus gilvus. Mycologia 87:805-820.

Rosewich UL, Pettway RE, McDonald BA, Kistler HC. 1999. High levels of gene flow and heterozygote excess characterize Rhizoctonia solani AG-1 IA (Thanatephorus cucumeris) from Texas. Fungal Genet Biol 28:148-159.

Saitou N, Nei M. 1987. The neighbor-joining method: a new method for reconstructing phylogenetic trees. Mol Biol Evol 4:406-425.

Shew HD, Main CE. 1985. Rhizoctonia leaf spot of fluecured tobacco in North Carolina. Plant Dis 69:901-903. , Melton TA. 1995. Target spot of tobacco. Plant Dis 79:6-11.

Stevens Johnk J, Jones RK, Shew HD, Carling DE. 1993. Characterization of populations of Rhizoctonia solani AG-3 from potato and tobacco. Phytopathology 83:854858.

Taylor JW, Jacobson DJ, Fisher MC. 1999. The evolution of asexual fungi: reproduction, speciation and classification. Annu Rev Phytopathol 37:197-246.

Vilgalys R, Gonzalez D. 1990. Ribosomal DNA restriction fragment length polymorphisms in Rhizoctonia solani. Phytopathology 80:151-158.

Vos P, Rogers R, Bleeker M, Reijans M, van de Lee T, Hornes M, Frijters A, Pot J, Peleman J, Kuiper M, Zabeau M. 1995. AFLP: a new technique for DNA fingerprinting. Nucl Acids Res 23:4407-4414.

Worrall JJ. 1997. Somatic incompatibility in basidiomycetes. Mycologia 89:24-36. 